\title{
Compressed sensing in optical coherence tomography
}

\author{
Nishant Mohan ${ }^{a}$, Ivana Stojanovic ${ }^{b}$, W. Clem Karl ${ }^{a, b}$, Bahaa E. A. Saleh ${ }^{b, c}$, and \\ Malvin C. Teich ${ }^{a, b}$ \\ ${ }^{a}$ Department of Biomedical Engineering, Boston University, Boston MA, USA; \\ ${ }^{b}$ Department of Electrical and Computer Engineering, Boston University, Boston MA, USA; \\ ${ }^{c}$ College of Optics and Photonics (CREOL), University of Central Florida, Orlando FL, USA
}

\begin{abstract}
Optical coherence tomography (OCT) is a valuable technique for non-invasive imaging in medicine and biology. In some applications, conventional time-domain OCT (TD-OCT) has been supplanted by spectral-domain OCT (SD-OCT); the latter uses an apparatus that contains no moving parts and can achieve orders of magnitude faster imaging. This enhancement comes at a cost, however: the CCD array detectors required for SD-OCT are more expensive than the simple photodiodes used in TD-OCT. We explore the possibility of extending the notion of compressed sensing (CS) to SD-OCT, potentially allowing the use of smaller detector arrays. CS techniques can yield accurate signal reconstructions from highly undersampled measurements, i.e., data sampled significantly below the Nyquist rate. The Fourier relationship between the measurements and the desired signal in SD-OCT makes it a good candidate for compressed sensing. Fourier measurements represent good linear projections for the compressed sensing of sparse point-like signals by random under-sampling of frequency-domain data, and axial scans in OCT are generally sparse in nature. This sparsity property has recently been used for the reduction of speckle in OCT images. We have carried out simulations to demonstrate the usefulness of compressed sensing for simplifying detection schemes in SD-OCT. In particular, we demonstrate the reconstruction of a sparse axial scan by using fewer than 10 percent of the measurements required by standard SD-OCT.
\end{abstract}

Keywords: Compressed sensing, optical coherence tomography

\section{INTRODUCTION}

Optical coherence tomography (OCT) is a valuable technique for non-invasive imaging in medicine and biology. ${ }^{1}$ In some applications, conventional time-domain OCT (TD-OCT) has been supplanted by spectral-domain OCT $(\mathrm{SD}-\mathrm{OCT}) ;^{2}$ the latter uses an apparatus that contains no moving parts and can achieve orders of magnitude faster imaging. This enhancement comes at a cost, however: the CCD array detectors required for SD-OCT are more expensive than the simple photodiodes used in TD-OCT.

In this work we explore the possibility of extending the notion of compressed sensing (CS) to SD-OCT, potentially allowing the use of smaller detector arrays. CS techniques can yield accurate signal reconstructions from highly under-sampled measurements, i.e., data sampled significantly below the Nyquist rate..$^{3,4}$ In particular, it has been shown that when the unknown signal is sparse, i.e., when it has a small proportion of nonzero contributions, CS techniques offer successful recovery with relatively few randomly under-sampled measurements. Moreover, CS techniques are applicable to linear sensing mechanisms in which the discrete sensing matrix enables incoherent sampling ${ }^{5}$ or when it satisfies the so-called restricted isometry property. ${ }^{6}$ The sparsity of the signal is then utilized to reduce the volume of collected data by employing nonlinear reconstruction that simultaneously enforces signal sparsity and consistency with the reduced data. Furthermore, it is known that Fourier measurements represent good linear projections for the compressed sensing of sparse point-like signals by random under-sampling of the frequency-domain data. ${ }^{4}$

\footnotetext{
Further author information: (Send correspondence to Nishant Mohan) Nishant Mohan: E-mail: nm82@bu.edu, Ivana Stojanovic: E-mail: ivanas@bu.edu, W. Clem Karl: Email: wckarl@bu.edu, Bahaa E. A. Saleh: Email: besaleh@creol.ucf.edu, Malvin C. Teich: Email: teich@bu.edu

Three-Dimensional and Multidimensional Microscopy: Image Acquisition and Processing XVII, edited by Jose-Angel Conchello, Carol J. Cogswell, Tony Wilson, Thomas G. Brown, Proc. of SPIE Vol. 7570, 75700L (C) 2010 SPIE · CCC code: $1605-7422 / 10 / \$ 18 \cdot$ doi: $10.1117 / 12.844217$

Proc. of SPIE Vol. $757075700 \mathrm{~L}-1$
} 
The Fourier relationship between the measurements and the desired signal in SD-OCT makes it a good candidate for compressed sensing. Additionally, axial scans in OCT are generally sparse in nature. This sparsity property was successfully used in recent work related to the reduction of speckle in OCT images. ${ }^{7}$

Sec. 2 provides a theoretical framework for the application of compressed sensing in OCT. In Sec. 3, we carry out simulations that demonstrate the potential usefulness of CS techniques in OCT. Finally, in Sec. 4 we discuss our results and mention some possible future work in this area.

\section{THEORY}

We first develop a linear-system model relating the discrete version of a physical object under observation, $\mathbf{x}$, and the discrete measured signal, $\mathbf{y}$. In our model, the physical object comprises a series scatterers with amplitude scattering coefficients $\alpha_{n}$. In SD-OCT, the spectral signal at a fixed transverse location of the sample is given by

$$
y(\nu) \propto S(\nu) \sum_{n} \alpha_{n} \cos \left(2 \pi d_{n} \nu / c\right),
$$

where $c$ is the speed of light in the medium, $\nu$ is the optical frequency, $S(\nu)$ is the spectrum of the light source, $d_{n}=n \Delta d$ represents the sampling grid, and $\alpha_{n}$ is zero everywhere except at the few locations where a scatterer exists. The DC component and cross terms in the SD-OCT signal have been excluded inasmuch as they are readily removed by the measurement technique reported in Ref. 8 . The measured signal in SD-OCT is the discrete version of $y(\nu)$, which we denote by $\mathbf{y}$. The axial scan is reconstructed by taking the DFT of this measured signal. As a result of the symmetry properties of the DFT, the first half of the transformed data represents the axial scan while the second half is simply the mirror image thereof. ${ }^{8}$ To conveniently use the DFT operator in a linear-system formulation, we therefore consider the first half of the object vector $\mathbf{x}$ to represent the series of scatterers with scattering coefficients $\alpha_{n}$ present at specific axial locations; the second half is, again, simply the mirror image.

Since the reconstruction of an axial scan is obtained by taking the DFT of the discrete measurement $\mathbf{y}$, it is represented by $\mathbf{F y}$, where $\mathbf{F}$ is the DFT matrix operator. Also, the reconstructed axial scan is, by definition, equivalent to $\mathbf{C x}$, where the operation by the matrix $\mathbf{C}$ results in a convolution of $\mathbf{x}$ with the point-spread function of the imaging system. We thus obtain the vector relation $\mathbf{F y}=\mathbf{C x}$ or $\mathbf{y}=\mathbf{F}^{-\mathbf{1}} \mathbf{C x}$. Defining $\mathbf{A}=\mathbf{F}^{-\mathbf{1}} \mathbf{C}$, we then have $\mathbf{y}=\mathbf{A x}$, representing a linear relationship between the measured signal and the object. In general, if the number of elements in $\mathbf{y}$ ( say $N$ ) is equal to that in $\mathbf{x}, \mathbf{A}$ is a square matrix and a solution to the linear system exists if $\mathbf{A}$ is a full-rank invertible matrix. If we have access to only a small set of elements of $\mathbf{y}$, the problem is defined as $\mathbf{y}_{\mathbf{k}}=\mathbf{A}_{\mathbf{k}} \mathbf{x}_{\mathbf{k}}$ where $\mathbf{y}_{\mathbf{k}}$ contains only a small number of elements of $\mathbf{y}$ (say $K$ ) and $\mathbf{A}_{\mathbf{k}}$ consists of the corresponding rows of the matrix operator $\mathbf{A}$.

Since $K \ll N$, such a system of equations is underdetermined and many solutions are possible. To overcome this difficulty, prior information about the signal $\mathbf{x}$ is incorporated into the reconstruction. When the signal $\mathbf{x}$ is sparse, an optimally sparse solution with only a few non-zero elements is sought. A direct formulation leads to the optimization problem: $\min _{\mathbf{x}}\|\mathbf{x}\|_{0}$ s.t. $\mathbf{y}_{\mathbf{k}}=\mathbf{A}_{\mathbf{k}} \mathbf{x}$, requiring an NP hard enumerative search. Instead, a computationally tractable formulation takes the form: $\min _{\mathbf{x}}\|\mathbf{x}\|_{1}$ s.t. $\mathbf{y}_{\mathbf{k}}=\mathbf{A}_{\mathbf{k}} \mathbf{x}$, where $l_{1}$ norm is a sparsity preserving convex relaxation of $l_{0}$ norm. This optimization problem is known in the literature as "basis pursuit". ${ }^{9}$ This formulation has also been motivated by the fact that under certain conditions on the observation matrix A, basis pursuit and the direct formulation have the same solution. ${ }^{10}$

Compressed sensing takes sparse reconstruction one step further by seeking to require as few measurements as possible while not compromising reconstruction quality. Let $T=\|\mathbf{x}\|_{0}$ denote the number of nonzero elements in $\mathbf{x}$. For the case when $\mathbf{C}$ is the identity matrix, it has been shown that the random under-sampling of $\mathbf{y}$ and the nonlinear reconstruction by basis pursuit lead to accurate reconstructions with high probability even when only $\mathcal{O}(T)$ measurements are obtained. In particular, given that the number of measurements satisfies

$$
K \geq G_{\delta} T \log (N),
$$

then with probability of at least $1-\mathcal{O}\left(N^{-\delta}\right)$, $\mathbf{x}$ can be reconstructed exactly as a solution of the $l_{1}$ minimization problem. Note that $G_{\delta}$ is typically a small constant, independent of $T$ and $N$, that depends on the desired 
(a)

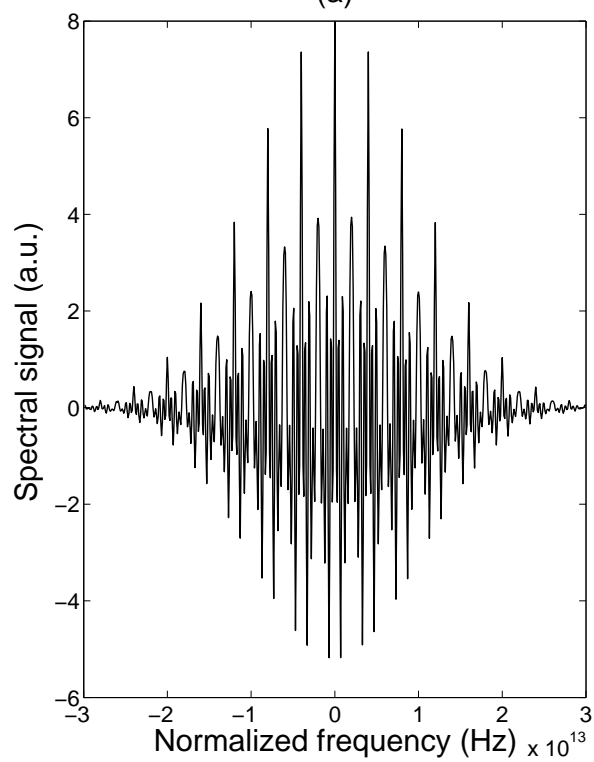

(b)

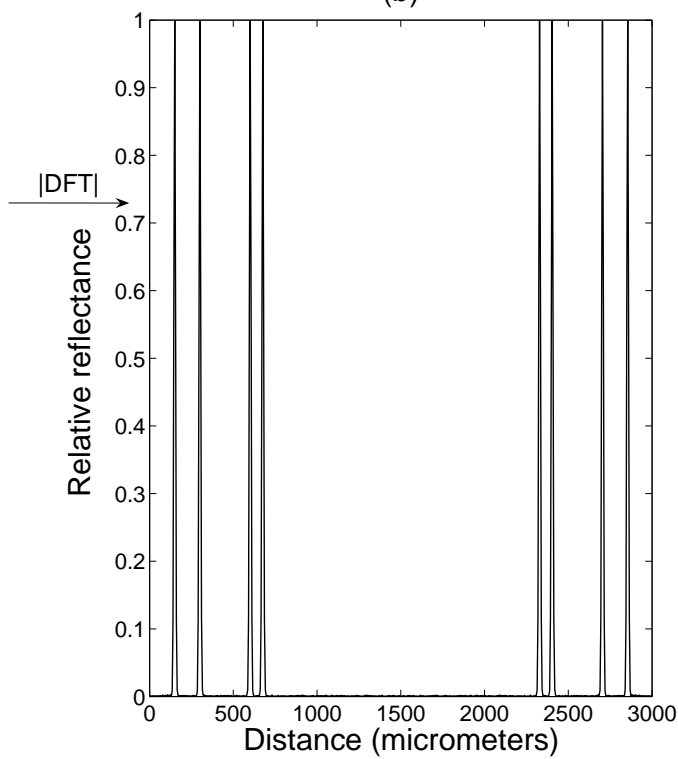

Figure 1. Simulation of a spectral signal in SD-OCT. Frequencies are normalized such that the central frequency corresponds to zero. (b) Axial OCT scan reconstructed by using the DFT.

accuracy parameter $\delta$. This result accommodates the reconstruction of any signal with a support of size $T$ and follows from a worst case analysis. In practice, a smaller number of measurements may be possible. ${ }^{4}$

Motivated by these insights, we empirically investigated the performance of the CS technique for the case when $\mathbf{C}$ is not the identity matrix, but rather a convolution operator corresponding to the axial point-spread function of the system, which is simply the Fourier transform of the source spectrum. We show in the next section that CS techniques can be employed in such a scenario. In the presence of noise more robust optimization methods are required. ${ }^{11,12}$ Therefore instead of the basis pursuit formulation we use the following unconstrained optimization method:

$$
\min _{\mathbf{x}}\left\|\mathbf{y}_{\mathbf{k}}-\mathbf{A}_{\mathbf{k}} \mathbf{x}_{\mathbf{k}}\right\|_{2}^{2}+\beta\left\|\mathbf{x}_{\mathbf{k}}\right\|_{1}
$$

where the regularization parameter $\beta$ determines the trade-off between data consistency and sparsity.

\section{SIMULATIONS AND RESULTS}

The measured data $\mathbf{y}$ is simulated using Eq. (1). The frequency $\nu$ is discretized with the difference between the samples being $\Delta \nu=10^{11} \mathrm{~Hz}$. In terms of wavelength, this corresponds to a measurement resolution of $\Delta \lambda=0.27 \mathrm{~nm}$ at $900 \mathrm{~nm}$. The source spectrum $S(\nu)$ is chosen to be a Gaussian function. Spectra of three different widths are used for these simulations, with standard deviations of $40 \Delta \nu, 120 \Delta \nu$, and $200 \Delta \nu$. Inasmuch as $\Delta \nu$ is kept constant for each of these cases, the broader spectra will have more sample points $(N=200,600$, and 1000, respectively). The axial location of the scatterers is chosen to be at $d_{n}=100,200,400,450$, and $500 \mu \mathrm{m}$. For four different object models, corresponding to $T=4,6,8$, and 10, non-zero scattering coefficients were created by choosing only the first $T / 2$ values of $d_{n}$ (the actual number of physical scatterers is $T / 2$ by virtue of the symmetry in the model discussed earlier). For simplicity, $\alpha_{n}$ is chosen to be constant for all $n$. White Gaussian random noise with a mean of zero and standard deviation of 0.01 is added to the simulated vector $\mathbf{y}$ to produce an SNR in the vicinity of $40 \mathrm{~dB}$. A convolution operator $\mathbf{C}$ is created that corresponds to the point-spread function of the system. Finally, the operator $\mathbf{A}$ is defined as $\mathbf{F}^{-\mathbf{1}} \mathbf{C}$. To demonstrate the possibility of compressed sensing, $\mathbf{y}_{\mathbf{k}}$ and $\mathbf{A}_{\mathbf{k}}$ are created by randomly choosing a small number of elements of $\mathbf{y}$ and the corresponding rows of $\mathbf{A}$. The estimate of the object $\mathbf{x}_{\mathbf{k}}$ is created by solving Eq. (3). The value of the regularization parameter $\beta=0.1$ gives satisfactory results for all of our cases. Note that the estimate of $\mathbf{x}_{\mathbf{k}}$ is, in 
(a)

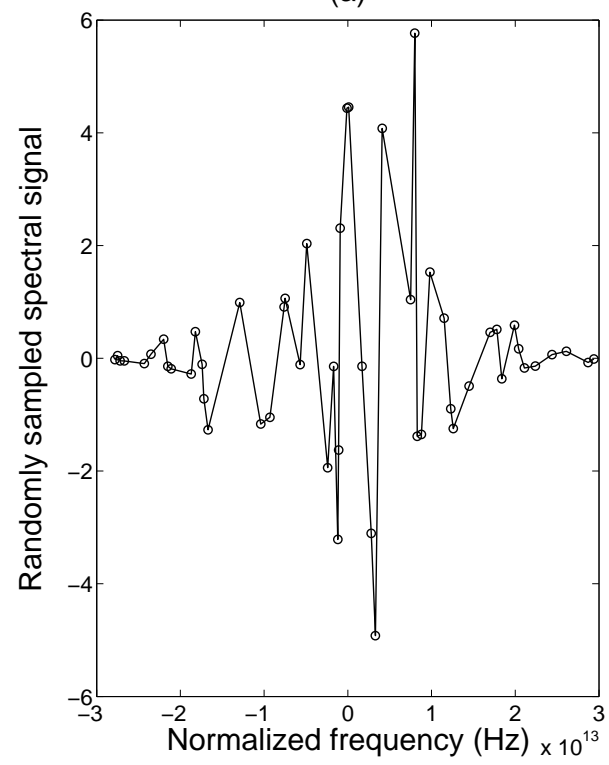

(b)

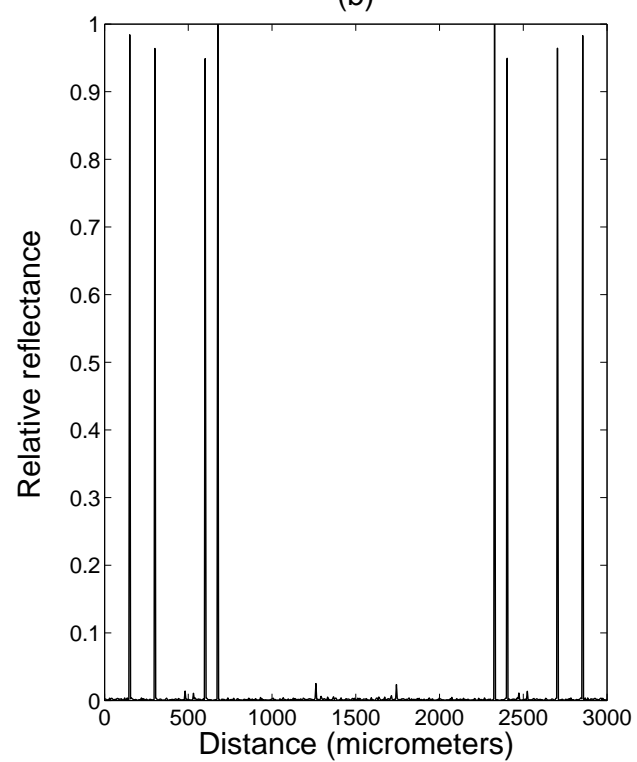

Figure 2. (a) Random selection of 52 of the 600 spectral-signal samples represented in Fig. 1(a). (b) Axial OCT scan reconstructed by solving the optimization problem specified in Eq. (3).

general, complex (as it is related to DFT of a real quantity); as in conventional SD-OCT we plot the magnitude of the resulting estimate for display. We are able to reconstruct the object for every case by using only $T \log (N)$ measurement samples, as suggested by Eq. (2). The location of the non-zero elements in the reconstructed vector are at the expected location in each case, but there is some variation in their amplitudes.

For the case of $T=8$ and $N=600$, we carried out Monte Carlo simulations by using 100 different sets of random elements of length $T \log (N) \sim 52$. The minimum mean square error per element for this case was found to be $2.34 \times 10^{-5}$. We demonstrate a reconstruction using $T=8$ and $N=600$ alongside a regular DFT based reconstruction. Figure 1 displays the reconstruction of the axial scan by direct Fourier transformation of the spectral data, using all of the data points, whereas Fig. 2 shows the reconstruction for the same object model with fewer than 10 percent of the measurements using compressed sensing. The similarity in the results is striking.

\section{DISCUSSION AND FUTURE WORK}

In this work we have demonstrated the possibility of using compressed sensing to simplify detection schemes in SD-OCT. This can potentially lead to the development of simpler SD-OCT systems that make use of smaller CCD arrays. We are currently investigating the limit on the minimum number of spectral measurements required with the imposition of sparsity conditions in SD-OCT. We also expect to consider the use of simpler sources for high-resolution OCT by extending the idea of using limited spectral data for reconstruction.

\section{ACKNOWLEDGMENTS}

This work was supported by the Bernard M. Gordon Center for Subsurface Sensing and Imaging Systems (CenSSIS), an NSF Engineering Research Center, and by a U.S. Army Research Office (ARO) Multidisciplinary University Research Initiative (MURI) Grant.

\section{REFERENCES}

[1] A. F. Fercher, W. Drexler, C. K. Hitzenberger, and T. Lasser, "Optical coherence tomography—principles and applications," Rep. Prog. Phys. 66, 239 (2003). 
[2] R. Leitgeb, C. Hitzenberger, and A. Fercher, "Performance of Fourier domain vs. time domain optical coherence tomography," Opt. Express 11, 889 (2003).

[3] D. L. Donoho, "Compressed sensing," IEEE Trans. Inform. Theory 52, 1289 (2006).

[4] E. J. Candes, J. Romberg, and T. Tao, "Robust uncertainty principles: Exact signal reconstruction from highly incomplete frequency information," IEEE Trans. Inform. Theory 52, 489 (2006).

[5] E. J. Candes and J. Romberg, "Sparsity and incoherence in compressive sampling," Inverse Problems 23, 969 (2007).

[6] E. J. Candes and T. Tao, "Decoding by linear programming," IEEE Trans. Inform. Theory 51, 4203 (2005).

[7] Z. Jian, Z. Yu, L. Yu, B. Rao, Z. Chen, and B. J. Tromberg, "Speckle attenuation in optical coherence tomography by curvelet shrinkage," Opt. Lett. 34, 1516 (2009).

[8] M. Wojtkowski, R. Leitgeb, A. Kowalczyk, and T. Bajraszewski, "In vivo human retinal imaging by Fourier domain optical coherence tomography," J. Biomed. Opt. 7, 457 (2002).

[9] S. S. Chen, D. L. Donoho, and M. A. Saunders, "Atomic decomposition by basis pursuit," SIAM J. Scientific Computing 20, 33 (1998).

[10] D. L. Donoho and M. Elad, "Optimally sparse representation in general (nonorthogonal) dictionaries via 11 minimization," Proc. Nat. Acad. Science 100, 2197 (2003).

[11] D. L. Donoho, M. Elad, and V. N. Temlyakov, "Stable recovery of sparse overcomplete representations in the presence of noise," IEEE Trans. Inform. Theory 52, 6 (2006).

[12] J. J. Fuchs, "Recovery of exact sparse representations in the presence of bounded noise," IEEE Trans. Inform. Theory, 51, 3601 (2005). 